\title{
Editorial
}

\section{Neurological Disease Burden in Geriatric Patients: Bangladesh Perspectives}

\author{
Mohammad Enayet HUSSAIN \\ Assistant Professor, Department of Neurology, National Institute of Neurosciences \& Hospital, Sher-E-Bangla Nagar, Agargaon, \\ Dhaka-1207, Bangladesh; Email: enayetdmc@yahoo.com; Cell no.: +8801716215871
}

The average age of the people of Bangladesh are increasing. The current data have shown that the average age of male and female are more than 70 years. Functional changes in the brain and nervous system are often the most marked of all the changes that occur in the human and in many cases there is no corresponding anatomical change. Lessened coordination, slowed afferent and efferent impulses, weakened and often decreased sensibility, impaired activity of the regulating centers and various forms of mental disturbance may be present, yet no morphological change can be found to account for them. These are called senile neuroses.

Some of the functional changes in the aged resemble the abnormal activities associated with disease in maturity. Senile tremor simulates paralysis agitans but the central canal of the cord may not be encroached upon as is the case in the diseased condition. On the other hand, extensive anatomical changes have been found without functional changes. Bunsen, who died at the age of eighty-nine, was engaged in profound scientific research up to the time of his death, yet his brain was greatly atrophied. The same condition was found in the brain of Mommsen, the great German historian. Among the earliest of the functional changes in the nervous system are delayed and weakened impulses. Action does not respond as rapidly to the will, and it requires a greater motor impulse to perform the act while greater mental concentration is required to obtain and hold sensory impressions. Brain fag sets in more rapidly than in maturity and while the quality of the work may not deteriorate, the amount of work that can be done at a time is less.

Aged writers who could write for ten or twelve hours without intermission during maturity must now take frequent rests else brain fag, then mental confusion and finally complete mental exhaustion set in. The rest must be either in the form of sleep or of some diversion which requires no mental exertion. When an old man falls asleep during a sermon or lecture, it is not through lack of interest but from brain fatigue following concentrated interest. Tendon reflexes are generally diminished. Sometimes the exaggerated tendon reflex is associated with tremor and a pronounced uncertain gait, the whole simulating cerebrospinal sclerosis. In such cases arteriosclerosis of the brain and cord are usually found and often cerebral softening. The functions of the sensory organs are impaired in old age. The sclerosis and flattening of the crystalline lens render accommodation for near objects difficult and presbyopia is produced. Where there has been myopia in earlier life it frequently happens that the senile flattening of the lens will so far reduce the former excessive convexity as to bring about a normal convexity of the emmetropic eye. This explains the so-called "second sight" of aged persons who had been obliged to use glasses in earlier life and can see well without glasses in old age.

The term "second sight" is also applied to a myopie condition that occurs in incipient cataract in the aged who have presbyopia or hypermetropia, seeing well at a distance but requiring convex lenses for reading. A swelling of the lens and an increase in its density during the formation of the cataract increases the refraction and the individual can now read without glasses but distance vision is impaired. The acuteness of vision is however not restored, but there is an increasing blurring and dimness depending upon the site, distribution and degree of opacity. Though rather frequent in the aged it is pathological. Weakened accommodation of the muscles interferes with motion of the organ. Owing to the weakening of the muscular fibers of the iris the pupils respond slowly to light and are generally contracted.

[Journal of Current and Advance Medical Research 2016;3(1):1] 